\title{
Prueba de ensayo de ácido húmico, para la producción de frijol castilla (Vigna unguiculata L.), en el campo experimental de los anitos provincia de Barranca
}

\author{
Test of human acid, for the production of castilla frijol (Vigna \\ unguiculata L.), in the experimental field of the anitos province of \\ Barranca
}

José Antonio Legua Cardenas ${ }^{1}$, Javier Jonás Espinoza García ${ }^{2}$, Dante Daniel Cruz Nieto $^{1}$, Francisco Espinoza Montesinos ${ }^{2}$, José del Carmen Ramírez Maldonado² Y Edgar Amador Espinoza Montesinos ${ }^{3}$

\section{RESUMEN}

La investigación se realizó en el campo experimental de Los Anitos y tiene como objetivo determinar las dosis de ácido húmico, en frijol castilla, se inició en noviembre del 2015 , se estableció los tratamientos $\left(\mathrm{T}_{1}=00, \mathrm{~T}_{2}=50, \mathrm{~T}_{3}=100, \mathrm{~T}_{4}=150 \mathrm{ml}\right.$ Liqhumus /20 1. agua), la aplicación se realizó en tres momentos, se evaluó hasta la cosecha y laboratorio. Este experimento se realizó en cuatro tratamientos y cuatro bloques, se empleó el Diseño de Bloque Completamente al Azar (DBCA). Su análisis estadístico que se operó fue el análisis de varianza al 1\%, 5\%, y la Prueba de Duncan al 5\%. Se obtuvo los resultados en que sobresalió la mayor dosis (150 ml/ 201 agua) el $\mathrm{T}_{4}$ con 48,075 cm de altura, 28,55 número de flores, 42,44 muestreo de número de vainas por planta, 103,63 g. muestreo de peso de vainas y 2,501 Tm/ ha rendimiento comercial, en laboratorio se precisó buenos resultado con: 18,35 cm de longitud de vaina, 12,67

\footnotetext{
1 Universidad Nacional José Faustino Sánchez Carrión. Huacho, Perú.

2 Universidad Nacional Santiago Antúnez de Mayolo. Huaraz, Perú.

3 Universidad para el Desarrollo Andino. Lircay-Huancavelica, Perú.

(C) Los autores. Este artículo es publicado por la Revista Aporte Santiaguino de la Universidad Nacional Santiago Antúnez de Mayolo. Este es un artículo de acceso abierto, distribuido bajo los términos de la Licencia Creative Commons Atribución-NoComercial-CompartirIgual 4.0 Internacional. (http://creativecommons.org/licenses/ by-nc-sa/4.0/), que permite el uso no comercial, distribución y reproducción en cualquier medio, siempre que la obra original sea debidamente citada.
} 
número de granos por vaina, 0,43 g peso de grano por vaina y 23,62 g sin embargo todos estuvieron en el rango de mediano $(18-25 \mathrm{~g})$. También sobresalió en mayor rentabilidad es de 45,92\%.

Palabras claves: Frijol Castilla; Ácido Húmico; Rendimiento; Rentabilidad

\begin{abstract}
The research was conducted in the Los Anitos experimental field and aims to determine the doses of humic acid, in castilla beans, started in November 2015, the treatments were established $(\mathrm{T} 1=00, \mathrm{~T} 2=50, \mathrm{~T} 3=100, \mathrm{~T} 4=150 \mathrm{ml}$ Liqhumus $/$ 201 . Water), the application was made in three moments, it was evaluated until harvest and laboratory. This experiment was carried out in four treatments and four blocks, using the Design of Block Completely Random (DBCA). The statistical analysis that was performed was the analysis of variance at $1 \%, 5 \%$, and Duncan's test at $5 \%$. The results were obtained in which the highest dose $(150 \mathrm{ml} / 201$ water $)$ stood out the T4 with 48,075 cm of height, 28,55 number of flowers, 42,44 sampling of number of pods per plant, 103,63 g. sampling of pod weight and 2,501 Tm / ha commercial yield, in laboratory good results were determined with: $18.35 \mathrm{~cm}$ of pod length, 12.67 number of grains per pod, 0,43 g grain weight per pod and 23,62 g, however, all were in the medium range $(18-25 \mathrm{~g})$. Also stood out in higher profitability is $45,92 \%$.
\end{abstract}

Keywords: Castilla Bean; Humic Acid; Yield; Profitability

\title{
INTRODUCCIÓN
}

El objetivo de la investigación fue determinar las diferentes pruebas de ensayos de ácido húmico, para el rendimiento del cultivo de frijol castilla en la Provincia de Barranca.

El frijol castilla es una leguminosa de alto valor nutricional de proteínas, calorías y carbohidrato, hierro, calcio, además de rápida cocción y fácil digestión Myfitnesspal (2016). Por estas propiedades alimenticias se está exportando a países como: Portugal, España, Grecia, Reino Unido Bélgica y Estados Unidos que son de mayor porcentaje. El Perú no es ajeno a este comercio; por esta razón se ha incrementado hectáreas de siembra como sucede en Piura Loreto y Lambayeque AREX (2014).

Por otro lado; Liqhumus, es una suspensión de humatos de potasio con un contenido mayor de $20 \%$ entre ácidos húmicos bio-activos y ácidos Fúlvicos. El producto es una enmienda húmica natural obtenida por la extracción alcalina de lignitos alemanes oxidados (conocidos como Leonardita) y presentada en forma de suspensión concentrada. Su aplicación incrementa la capacidad de intercambio catiónico y la fertilidad del suelo (SOLAGRO, 2016). 
Gilliavod (2000), expone que a pesar del considerable progreso experimentado por la química del humus en los últimos veinte años, la estructura de la materia orgánica del suelo continúa sin ser conocida en su mayor parte. En la actualidad se asiste al desarrollo creciente de investigación que se encuentra limitadas por la aplicación generalizada de planteamientos procedentes de la química de la ligninina y carbon.

Pinedo (2012), menciona que el $\mathrm{T}_{4}$ (50 Lt./ Ha aplicados $50 \%$ a la siembra y $50 \% 15$ dds (días después de la siembra) con promedio más alto de $32302,92 \mathrm{~kg} /$ ha, superó estadísticamente a los demás tratamientos, seguido del $\mathrm{T}_{3}(30 \mathrm{Lt} / \mathrm{ha}$ aplicados $50 \%$ a la siembra y $50 \% 15 \mathrm{dds}), \mathrm{T}_{2}\left(200 \mathrm{~kg} / \mathrm{ha}-100 \%\right.$ aplicados a la siembra), $\mathrm{T}_{1}(150$ $\mathrm{kg} / \mathrm{Ha}-100 \%$ aplicados a la siembra) y $\mathrm{T}_{0}$ (testigo) quienes alcanzaron los promedios de 26 447,92 Kg/ Ha, $25045.50 \mathrm{Kg} / \mathrm{Ha}, 22$ 685,83 Kg/ Ha y 15 110,42 Kg/ ha de rendimiento.

Estévez (2006), expone que la dosis que se utilizó para cada producto fue de D1 (1 Lt/ ha), D2 (1,5 Lt / ha) y D3 (2 Lt/ha). Dicho estas dosis se observa en el siguiente, los resultados que muestra que se obtuvo buen rendimiento con las dosis de aplicación con el P1 (Eco- Hum DX) con dosis de D2 (1 Lt /HA) 38,446 kg/ tratamiento), frente Codahumus (P2), Acido Húmico (P3).

Obando (2005), manifiesta en su tesis la cantidad de vainas por racimo floral precisa que la mayor cantidad se obtuvo en el T6 con 28,98, seguido del T5 con 28,47, T4 con 28,03 T3 con 26,69 T2 con 25,75, T1 con 24,12 Tn/ha. Asimismo alcanzó mayor rentabilidad económico el tratamiento 6 (2,25 Lt / ha de Eco Hum Dx). Por tal motivo se recomienda ésta dosis para el cultivo de vainita.

López (2013), concluye en sus resultados que son más sobresaliente el $\mathrm{T}_{2}(200 \mathrm{~kg} / \mathrm{ha})$ con 1028,84 kg/ha y $\mathrm{T}_{3}\left(300 \mathrm{~kg} / \mathrm{Ha}\right.$ ) con 1026,63 kg/ha y menor rendimiento $\mathrm{T}_{1}(100$ $\mathrm{kg} / \mathrm{ha}$ ) con $978,77 \mathrm{Kg} / \mathrm{Ha}$ y T0 con $872,1 \mathrm{~kg} / \mathrm{ha}$. Lo cual indica que el $\mathrm{T}_{2}$ obtuvo buen rendimiento con 1028,84 kg/ha en referente a la mayor dosis de $\mathrm{T}_{3} \mathrm{y}$ menor dosis $\mathrm{T}_{1}, \mathrm{~T}_{0}$.

Mencionado estos lugares, se puede afirmar que nuestra provincia se viene sembrando cada vez más extensas área. Por esta razón se realizó este experimento, lo cual se utilizó abono natural por su concentración de ácido Húmico (Liqhumus), que es un producto que contiene $20 \%$, que se aplica en la base de la planta; ya que mejora la condiciones químicas y biológicas del suelo disponibilidad de nutrientes. Cabe indicar que el manejo agronómico fue igual para todos; solo cambio las dosis $\mathrm{T} 1=00, \mathrm{~T} 2=50, \mathrm{~T} 3=100$, T4 $=150 \mathrm{ml} / 201$ agua. Dicho experimento se realizó en el Sector los Anitos, Distrito de Barranca, sembrándose el 12 de noviembre del 2015, por lo que se evaluó los parámetros de evaluación, obteniéndose los promedios de cada variable. 


\section{MATERIALES Y MÉTODOS}

En este proceso se realizó las aplicaciones adecuadas durante el desarrollo del cultivo lo cual consistió en una investigación descriptiva que describe los resultados de las evaluaciones y comparativa que compara y contrasta los tratamientos de dosis con otros autores. La metodología se muestra en la tabla 1.

Tabla 1. Aplicación metodológica, de acuerdo a su etapa de evaluación

\begin{tabular}{|c|c|c|}
\hline \multirow{2}{*}{$\begin{array}{l}\text { Etapa de } \\
\text { evaluación }\end{array}$} & \multicolumn{2}{|c|}{ Aplicación metodológica } \\
\hline & Descriptiva & Comparativo \\
\hline Etapa vegetativa & $\begin{array}{l}\text { Se evaluó los resultados de las } \\
\text { aplicaciones de dosis en cada } \\
\text { parcela durante toda esta etapa, } \\
\text { seguido se llevó una estadística. }\end{array}$ & $\begin{array}{l}\text { En este proceso se realizó las } \\
\text { comparaciones de los resultados } \\
\text { mediante toma de datos de cada } \\
\text { variable durante dicha etapa. }\end{array}$ \\
\hline $\begin{array}{c}\text { Etapa reproduc- } \\
\text { tiva }\end{array}$ & $\begin{array}{l}\text { Consistió en tomar los prome- } \\
\text { dios de vainas por planta median- } \\
\text { te el muestreo, a fin de conocer } \\
\text { sus características. }\end{array}$ & $\begin{array}{l}\text { En esta etapa se hizo evaluacio- } \\
\text { nes y se llevó a cuadros estadís- } \\
\text { ticos, con el fin de comparar los } \\
\text { resultados. }\end{array}$ \\
\hline Rendimiento & $\begin{array}{l}\text { Aquí se precisó los efectos de las } \\
\text { dosis de ácido húmico, en cada } \\
\text { parcela, evaluándose las variables } \\
\text { de rendimiento. }\end{array}$ & $\begin{array}{l}\text { Los resultados se tomaron a base } \\
\text { de promedios de cada parcela. } \\
\text { Se determinó los efectos de dosis } \\
\text { de ácido húmico. }\end{array}$ \\
\hline
\end{tabular}

\section{Análisis estadísticos}

Los datos estadísticos, para el procesamiento del análisis estadístico fueron: El análisis de varianza y la Prueba de Duncan.

\section{Análisis de varianza (ANVA)}

Se aplicó el análisis de varianza (ANVA $1 \%$ y $5 \%$ ) tabla 2; este se adecua a la comparación de tratamiento en la aplicación de dosis de ácido húmico. Mediante esta comparación permitió conocer el nivel de significativo o no significativo. 
Prueba de ensayo de ácido húmico, para la producción de frijol castilla (Vigna unguiculata L.)||

Tabla 2. Análisis de varianza que se utilizó en la investigación

\begin{tabular}{|c|c|c|c|c|c|c|}
\hline $\begin{array}{l}\text { Fuente de } \\
\text { Variación }\end{array}$ & SC & G1 & $\mathrm{CM}$ & $\begin{array}{l}\text { Modelo I } \\
\text { E(CM) }\end{array}$ & $\begin{array}{l}\text { Mod- } \\
\text { elo II } \\
\text { E(CM) }\end{array}$ & F. cal \\
\hline Bloques & $\mathrm{SC}_{\mathrm{b}}$ & $b-1$ & $C M_{b}=\frac{S_{b}}{(b-1)}$ & $\frac{\sigma^{2}+\sum \beta_{j}^{2}}{(b-1)}$ & $\sigma_{\mathrm{e}}^{2}+t \sigma_{\beta}^{2}$ & $\frac{\mathrm{CM}_{\mathrm{b}}}{\mathrm{CM}_{\mathrm{e}}}$ \\
\hline Tratamiento & $\mathrm{SC}_{\mathrm{tr}}$ & $t-1$ & $C \mathrm{M}_{\mathrm{tr}}=\frac{\mathrm{SC}_{\mathrm{tr}}}{(\mathrm{t}-1)}$ & $\frac{\sigma_{\mathrm{e}}^{2}+\mathrm{b} \sum \mathrm{t}_{\mathrm{j}}^{2}}{(\mathrm{t}-1)}$ & $\sigma_{\mathrm{e}}^{2}+\mathrm{b} \sigma_{\mathrm{t}}^{2}$ & $\frac{\mathrm{CM}_{\mathrm{tr}}}{\mathrm{CM}_{\mathrm{e}}}$ \\
\hline Error & $\mathrm{SC}_{\mathrm{e}}$ & $(b-1)(t-1)$ & $C \mathrm{M}_{\mathrm{e}}=\frac{\mathrm{SC}_{\mathrm{e}}}{(\mathrm{b}-1)(\mathrm{t}-1)}$ & $\sigma_{\mathrm{e}}^{2}$ & $\sigma_{\mathrm{e}}^{2}$ & \\
\hline Total & $\mathrm{SC}_{\mathrm{t}}$ & bt -1 & & & & \\
\hline
\end{tabular}

Fuente: Lind y et al. (2008), "Estadística Aplicada a los Negocios y la Economía

\section{Prueba de comparación múltiple}

En cuanto a la comparación de los tratamientos cuantitativamente se utilizó la prueba de Duncan al $5 \%$ de error, ya que esta operación permitió comparar que dosis fue adecuado en los parámetros de evaluación.

\section{Tratamientos de investigación}

A continuación se muestra la tabla 3, en el cual muestra los tratamientos que se aplicaron las dosis de Ácido húmico (Liqhumus) en tres momentos de aplicación 10, 25 y 45 días después de la siembra de frijol castilla.

Tabla 3. Dosis de aplicación de ácido húmico, en función a los tratamientos

\begin{tabular}{ccc}
\hline \multirow{2}{*}{ Tratamiento } & \multicolumn{2}{c}{ Dosis de aplicación de A. húmico } \\
\cline { 2 - 3 } & Dosis (m1/20 1. Agua) & Dosis (1./cilindro) \\
\hline T1 & 0.0 & 0,0 \\
T2 & 50 & 0,5 \\
T3 & 100 & 1,0 \\
T4 & 150 & 1,5 \\
\hline
\end{tabular}

Nota: Es importante que se tuvo en cuenta el manejo agronómico como: Labores culturales y sanidad vegetal. 


\section{RESULTADOS Y DISCUSIÓN}

\section{Altura de planta de frijol castilla}

La tabla 4, indica queno tuvo efecto de dosis de ácido húmico (Liqhumus); esto significa que los tratamientos y bloques no son significativos. Su coeficiente de variación que se determinó fue de 7,49\%, ligera variación depromedios.

Tabla 4. Análisis varianza de altura de planta

\begin{tabular}{|c|c|c|c|c|c|c|c|}
\hline \multirow[b]{2}{*}{ F. V } & \multirow[b]{2}{*}{ G.L. } & \multirow[b]{2}{*}{ S.C. } & \multirow[b]{2}{*}{ C.M. } & \multirow[b]{2}{*}{ F.C. } & \multicolumn{2}{|c|}{ F.T } & \multirow[b]{2}{*}{ Sifgn. } \\
\hline & & & & & 0.05 & 0.01 & \\
\hline Bloque & 3 & 3,706 & 1,23 & 0,11 & 3,86 & 6,99 & N.S. \\
\hline Tratamiento & 3 & 51,07 & 17,0 & 1,45 & 3,86 & 6,99 & N.S. \\
\hline Error & 9 & 105,68 & 11,7 & & & & \\
\hline Total & 15 & 160,46 & & & & & \\
\hline
\end{tabular}

En la figura 1, se expone que la mayor altura alcanza el T4 con 48,08 cm, dicho resultado se fundamenta con Estévez (2006), menciona en su tesis acerca de la aplicación de tres dosis ácidos húmicos comerciales en el cultivo de brócoli, se determinó que la aplicación de (P1) Eco Hum Dx con dosis de (D3) 2 Lt/ ha se obtuvo mayor altura con 3,914 cm., (P1) Eco hum Dx con dosis de (D2) 1,5 Lt/ha, 3,824 cm, y (P1) $(\mathrm{D} 1=1 \mathrm{Lt} / \mathrm{ha}) 3,8 \mathrm{~cm}$

Según Díaz (2015), precisa que los productos que contienen ácido húmico en especial el foliar Extra follaje 36-6-10, alcanzo mayor altura de planta con respecto a los demás tratamientos, pues la diferentes de este producto con el Extra follaje Extra, tiene más dosis de nitrógeno que sobresale en altura de planta que alcanzó a 1,15 m. 


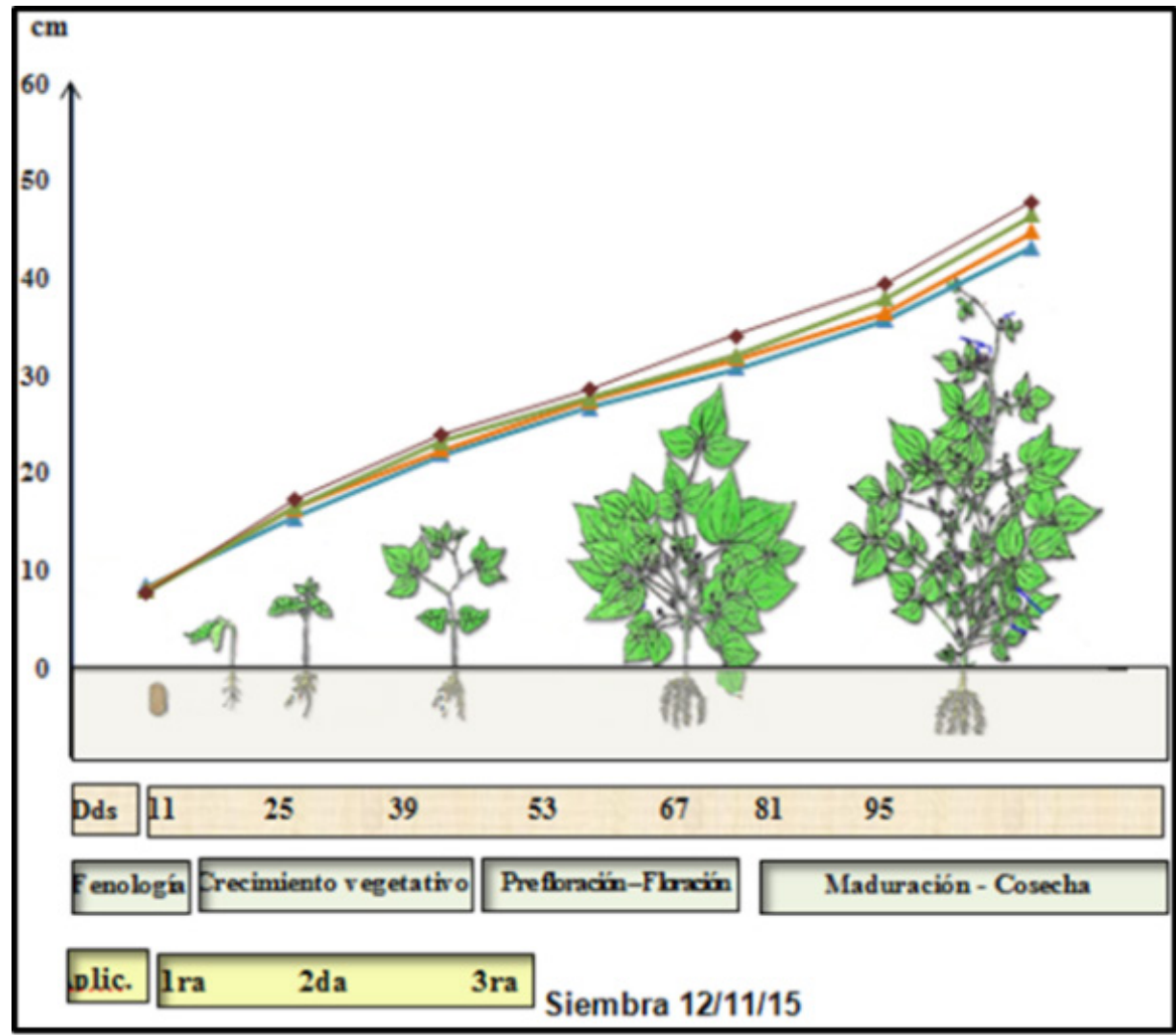

\begin{tabular}{|cccccc|}
\hline \multicolumn{6}{c}{ Tratamiento (cm.) } \\
\hline $\begin{array}{c}\text { F. } \\
\text { evaluación }\end{array}$ & D.d.s. & T1 $\square$ & T2 $\square$ & T3 & T4 \\
\hline $22 / 11 / 15$ & 11 & 8.5 & 8.13 & 7.9 & 7.8 \\
$06 / 12 / 15$ & 25 & 15.5 & 16.5 & 16.6 & 17.4 \\
$20 / 12 / 15$ & 39 & 21.98 & 22.5 & 23.28 & 23.9 \\
$03 / 01 / 16$ & 53 & 26.75 & 27.5 & 27.75 & 28.75 \\
$17 / 01 / 16$ & 67 & 30.75 & 31.75 & 32.25 & 34.25 \\
$31 / 01 / 16$ & 81 & 35.75 & 36.5 & 38 & 39.5 \\
$14 / 02 / 16$ & 95 & 43.33 & 44.83 & 46.55 & 48.08 \\
\hline
\end{tabular}

Figura 1. Altura de planta según dosis

\section{Número de flores por planta}

Según la Prueba de Duncan, se observa la table 5 que el $\mathrm{T}_{4}$ alcanzó mayor cantidad de flores por planta de 28,55; después del $\mathrm{T}_{3} \operatorname{con} 26,99$ Flores, $\mathrm{T}_{2}$ con 25,74 flores y por último el $\mathrm{T}_{1}$ con 24,79 flores. Por lo que se determina que a esta dosis se obtuvo buena cantidad significativa en todos los tratamientos a los 54 días después de la siembra. 
Tabla 5. Prueba de Duncan de Número de flores por planta

\begin{tabular}{cccc}
\hline Tratamiento & Dosis $(\mathrm{ml} . / 20 \mathrm{l})$. & Resultados $\left(\mathrm{N}^{\circ}\right)$ & Nivel \\
\hline $\mathrm{T}_{4}$ & $\mathbf{1 5 0}$ & 28,55 & $\mathbf{a}$ \\
$\mathrm{T}_{3}$ & $\mathbf{1 0 0}$ & 26,99 & $\mathbf{a}$ \\
$\mathrm{T}_{2}$ & $\mathbf{5 0}$ & 25,74 & $\mathbf{a}$ \\
$\mathrm{T}_{1}$ & $\mathbf{0 0}$ & 24,79 & $\mathbf{a}$ \\
\hline
\end{tabular}

Nota: Letras iguales en el mismo nivel son estadísticamente similares en sus resultados

\section{Número de vainas de Madurez fisiológica}

Los resultados de la prueba de Duncan en la tabla 6 indican que la mayor cantidad de vainas es el $\mathrm{T}_{4}$ con 20,6 siendo estadísticamente similar al $\mathrm{T}_{3}$ con 17,87 vainas y $\mathrm{T}_{2}$ con 16,37 vainas en (a y b) y diferente al de menor cantidad de $T_{1}$ con 13,06 vainas (b).

Tabla 6. Prueba de Duncan de $\mathrm{N}^{\circ} 6$ de vainas de Madurez Fisiológica

\begin{tabular}{ccccc}
\hline Tratamiento & Dosis $(\mathrm{ml} . / 20 \mathrm{l})$. & Resultados $\left(\mathrm{N}^{\circ}\right)$ & \multicolumn{2}{c}{ Nivel } \\
\hline $\mathrm{T}_{4}$ & $\mathbf{1 5 0}$ & 20,60 & $\mathbf{a}$ & $\mathbf{b}$ \\
$\mathrm{T}_{3}$ & $\mathbf{1 0 0}$ & 17,87 & $\mathbf{a}$ & $\mathbf{b}$ \\
$\mathrm{T}_{2}$ & $\mathbf{5 0}$ & 16,37 & $\mathbf{a}$ & $\mathbf{b}$ \\
$\mathrm{T}_{1}$ & $\mathbf{0 0}$ & 13,06 & & $\mathbf{b}$ \\
\hline
\end{tabular}

Nota: Letras diferentes en el mismo nivel son estadísticamente diferentes en los resultados.

\section{Muestreo de número de vainas por planta}

Las estadísticas obtenidas mediante la prueba de Duncan, tabla 7 , determinó que el $\mathrm{T}_{4}$ con mayor dosis obtiene 42,44 vainas, en comparación al $\mathrm{T}_{3}$ con 40,09; $\mathrm{T}_{2} \operatorname{con} 38,875$ y el de menor cantidad el $\mathrm{T}_{1}$ con 37,47 vainas por planta. 
Tabla 7. Prueba de Duncan de Muestreo de Número de vainas por planta.

\begin{tabular}{cccc}
\hline Tratamiento & Dosis $(\mathrm{ml} . / 20 \mathrm{l})$. & Resultados $\left(\mathrm{N}^{\circ}.\right)$ & Nivel \\
\hline $\mathrm{T}_{4}$ & $\mathbf{1 5 0}$ & 42,438 & $\mathbf{a}$ \\
$\mathrm{T}_{3}$ & $\mathbf{1 0 0}$ & 40,095 & $\mathbf{a}$ \\
$\mathrm{T}_{2}$ & $\mathbf{5 0}$ & 38,875 & $\mathbf{a}$ \\
$\mathrm{T}_{1}$ & $\mathbf{0 0}$ & 37,470 & $\mathbf{a}$ \\
\hline
\end{tabular}

Nota: Letras diferentes en el mismo nivel son estadísticamente diferentes en los resultados.

\section{Muestreo de peso de vainas por planta}

El análisis a continuación se realizó a los 103 días después de la siembra. Lo cual se tomó los datos de peso de vainas por planta. Esto se muestra en la tabla 8, que precia el $\mathrm{T}_{4}$ obtuvo el mayor peso con 103,63 g diferenciando se de $\mathrm{T}_{3} \operatorname{con} 97,66 \mathrm{~g}$; $\mathrm{T}_{2}$ con 91,16 g y el de menor cantidad $\mathrm{T}_{1}$ con 87,63 g. Además todos son homogéneos (a)

Tabla 8: Prueba de Duncan de muestreo de peso de vainas por planta

\begin{tabular}{cccc}
\hline Tratamiento & Dosis (ml. / 20 1.) & Resultados (g.) & Nivel \\
\hline $\mathrm{T}_{4}$ & $\mathbf{1 5 0}$ & 103,63 & $\mathbf{a}$ \\
$\mathrm{T}_{3}$ & $\mathbf{1 0 0}$ & 97,66 & $\mathbf{a}$ \\
$\mathrm{T}_{2}$ & $\mathbf{5 0}$ & 91,16 & $\mathbf{a}$ \\
$\mathrm{T}_{1}$ & $\mathbf{0 0}$ & 87,63 & $\mathbf{a}$ \\
\hline
\end{tabular}

Fuente de información: El Autor (2016)

\section{Rendimiento comercial por hectárea}

En la tabla 9, se observa que el $\mathrm{T}_{4}$ obtuvo mayor rendimiento con 2,50 th/ ha, en comparación a los demás resultados se puede relacionar con el aumento de dosis. También se puede afirmar que todos de un mismo nivel (a), siendo homogéneos. 
| José Legua, Javier Espinoza, Dante Cruz, Francisco Espinoza, José del Carmen Ramírez y Edgar Espinoza

Tabla 9. Prueba de Duncan del rendimiento comercial

\begin{tabular}{cccc}
\hline Tratamiento & Dosis (ml. / 20 l.) & Resultados (Tm /Ha) & Nivel \\
\hline $\mathrm{T}_{4}$ & $\mathbf{1 5 0}$ & 2.5013 & $\mathbf{a}$ \\
$\mathrm{T}_{3}$ & $\mathbf{1 0 0}$ & 2.2516 & $\mathbf{a}$ \\
$\mathrm{T}_{2}$ & $\mathbf{5 0}$ & 2.1505 & $\mathbf{a}$ \\
$\mathrm{T}_{1}$ & $\mathbf{0 0}$ & 2.0044 & $\mathbf{a}$ \\
\hline
\end{tabular}

Fuente de información: El Autor (2016)

\section{Análisis de rentabilidad}

Se expone el análisis económico en la figura 2, que tiene la tendencia lineal, es decir va aumentando con relación a la dosis, es decir la ganancia es mayor en $\mathrm{T}_{4}$ con 0,45 Nuevos soles. También se afirma que ha esta dosis mayor puede generar mayor beneficio.

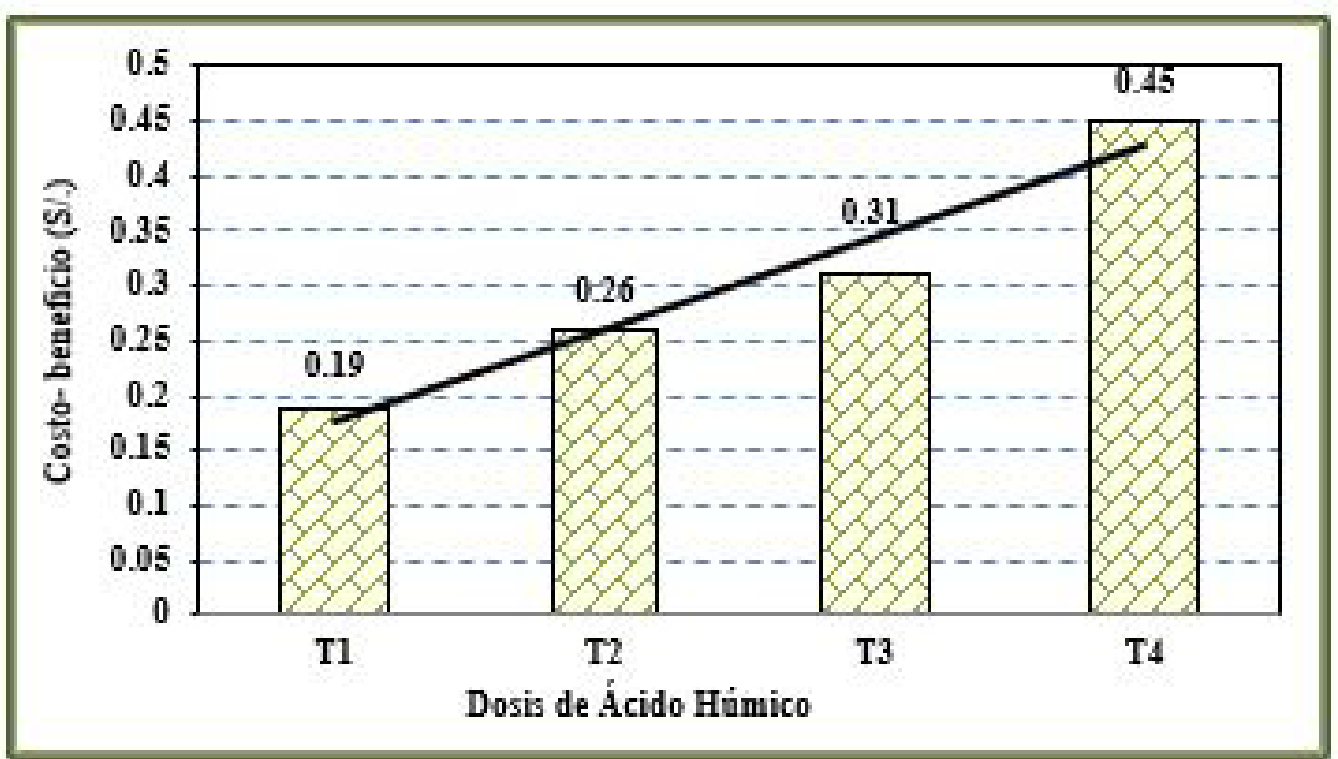

Figura 2. Análisis de rentabilidad, de las dosis de Ácido Húmico 


\section{CONCLUSIONES}

De acuerdo a los parámetros de evaluación, se determinó las características físicas; obteniéndose buenos resultados a mayor dosis en: altura de planta, número de flores por planta, número de vainas de madurez fisiológica, muestro de número y peso de vainas por planta, rendimiento total por parcela y comercial.

En cuanto a las medidas y peso en laboratorio se obtuvo buenos resultados en: longitud de vaina, tamaño de grano por tratamiento, número de granos por vaina y, peso de granos.

Cabe indicar que ha mayor dosis de ácido húmico (Liqhumus), se obtuvo buen rendimiento, siendo el $\mathrm{T}_{4}$ con 2,5013 $\mathrm{Tm} / \mathrm{h}$, lo cual precisa que se obtuvo buena utilidad económica, el mayor costo beneficio con S/ 0,46 Nuevos Soles.

También se apreció que el $\mathrm{T}_{4}$ obtuvo buena presencia en vigor, suculencia, resistencia problemas fitosanitarios; ya sea por la influencia del desarrollo biológico en el suelo obteniéndose buen rendimiento y calidad de vaina.

\section{RECOMENDACIONES}

Se debe realizar investigaciones acerca de mayor dosis de ácido húmico (Liqhumus) con otros cultivos de leguminosas; puesto que a dicha dosis obtuvo buenos resultados en rendimiento, calidad de vaina por planta y mayor rentabilidad económica.

Es importante usar semilla certificada tener en cuenta la historia fitosanitario y utilizar productos naturales como ácido húmico; ya que mejora las condiciones, químicas y biológicas en el suelo.

\section{REFERENCIAS BIBLIOGRÁFICAS}

AREX. 2014. Asociación Regional de Exportadores Lambayeque. "Perfil comercial de frijol castilla”. Área de comercio exterior. Lambayeque - Perú.

Díaz, A. 2015. "Efecto de cuatro Fertilizantes Foliares Inorgánicos sobre las características Agronómicas y Rendimiento del Pasto Panicum máximum cultivar Tanzania en Zungarococha - Iquitos - Loreto". Tesis para optar el título Profesional de Ingeniero Agrónomo. Iquitos-Perú.

Estévez, V. 2006. "Efectos de la aplicación de tres ácidos húmicos comerciales con diferentes dosis en el cultivo de brócoli (Brassica oleracea var. Italica) en la hacienda Pastaví, Canton Otavalo, Parroquia Quichinche”. Tesis para optar el Título Profesional de Ingeniero Agropecuario. 
Gilliavod, N. 2004. "Formación Del Humus". < http://infomorelos.com/ecologia/ humus.html> [Consulta: 09-10-2017]

Lind, D.; Marchal, W. y Wathen, S. 2008. "Estadística Aplicada a los Negocios y la Economía”. Coastal Carolina University and University of Toledo - EE.UU. Traducción de: Jorge Yescas y Javier León Cárdenas- México. 428.

SOLTAGRO. 2016. "Solagro: Soluciones técnicas del agro S.A.C.”. < http:/ / soltagro. com/productos/acidos-humicos/details/1/92.html > [Consulta: 09-08-2015]

López, T. 2013. “Dosis de Ácido Húmico granulado de Leonardita en el cultivo de Col China (Brassica pekinensis) Variedad Kiboho 90 F-1, Sector Quillo Allpa - Distrito y Provincia de Lamas". Tesis para optar el título profesional de Ingeniero Agrónomo. Universidad Nacional de San Martín. Tarapoto - Perú.

Myfitnesspal. 2016. "Calorías en Verificado Frijol Castilla” Información nutricional. Verificado - Frijol Castilla. <http://www.myfitnesspal.com/es/food/cal ories/ verificado-frijol-castilla- 374406207?v2=false $>$ [Consulta: 29-08-2015]

Obando, E. 2005. "Efecto de 6 niveles de Eco Hum dx® en el rendimiento de vainita (Phaseolus vulgaris) bajo invernadero". Tesis para optar el título profesional de Ingeniero Agropecuario. Universidad Católica del Ecuador.

Pinedo, D. 2012. "Dosis de ácido húmico granulado de Leonardita y ácidos húmicos y fúlvicos con macro y micro elementos en el cultivo de lechuga (Lactuca sativa L.) Variedad Great Lakes 659, bajo condiciones agroecológicas en la Provincia de lamas”. Tesis para optar el Título Profesional de Ingeniero Agrónomo. Tarapoto - Perú.

Recepción: 17/11/2018

Aceptación: 20/12/2018

\section{Correspondencia}

José Antonio Legua Cardenas

jose_legua@yahoo.es 